\title{
NDP kinases, développement et cancer : une action via des protéines liant le GTP?
}

Les nucléosides diphosphate (NDP) kinases sont des enzymes ubiquitaires, essentielles pour la synthèse des nucléosides triphosphate, en particulier le GTP. L'identification, comme NDP kinases, des produits de deux gènes, l'un awd, impliqué dans le développement de la drosophile et l'autre, nm23, décrit chez les mammifères comme un suppresseur du potentiel métastasique de cellules tumorales, suscite un regain d'intérêt pour l'étude de ces enzymes, jusqu'ici considérées uniquement comme éléments du métabolisme intermédiaire. Un rôle des NDP kinases dans la prolifération tumorale ou le développement est peut-être à rechercher dans leur association avec des protéines liant le GTP, dont elles pourraient faciliter la conversion en leur forme activée liée au GTP.

\section{Marie-Lise Lacombe Ioan Lascu Xavier Sastre-Garau Valérie Wallet Michel Véron}

\section{ADRESSES}

M.-L. Lacombe : chargée de recherche au Cnrs. I. Lascu : docteur ès sciences, boursier de la Ligue nationale contre le cancer.

V. Wallet : étudiante en doctorat, université de Paris.

M. Véron: directeur de recherche au Cnrs. Unité de biochimie cellulaire, Institut Pasteur. 28 rue du Docteur-Roux, 75724 Paris Cedex 15, France.

$\mathrm{X}$. Sastre-Garau : docteur en médecine, médecin hospitalier. Laboratoire de pathologie, Institut Curie, 26, rue d'Ulm, 75240 Paris a séquence d'événements caractérisant la transformation maligne des cellules est loin d'être connue précisément. Bien que les travaux de Warburg aient montré, déjà dans les années vingt, que le métabolisme de la cellule tumorale est grandement altéré, les recherches sur les enzymes du métabolisme furent quelque peu délaissées ces dernières années, au profit des études concernant les oncogènes. Des travaux menés conjointement aux États-Unis sur des cellules tumorales et sur la drosophile [1], en France et en Allemagne sur un eucaryote primitif, l'amibe Dictyostelium discoideum [2, 3], indiquent qu'une enzyme clé de la synthèse des nucléotides, la nucléoside diphosphate kinase (NDP kinase) est impliquée dans le développement embryonnaire et la prolifération tumorale $[3,4]$.
L'objet de cette revue est de faire le point sur ces données récentes dans la perspective particulière d'un rôle des NDP kinases dans l'activation des protéines liant les nucléotides guanyliques.

\section{Les NDP kinases}

Bien que les NDP kinases (EC 2.7.4.6.) aient été découvertes dès 1953, ce n'est qu'en 1990 que furent déterminées les premières structures primaires [2, 5, 6]. Ce sont des enzymes ubiquitaires responsables de la synthèse des nucléosides triphosphates à partir des nucléosides diphosphates correspondants (pour une revue : [7]), qui constituent un lien essentiel entre la formation de l'ATP par la voie de la phosphorylation oxydative et la synthèse des autres nucléosides triphosphates (figure 1). Le 
mécanisme réactionnel des NDP kinases est de type Ping-Pong avec formation d'un intermédiaire phosphorylé sur un résidu histidine.

\section{$\mathrm{N}_{1} \mathrm{TP}+\mathrm{N}_{2} \mathrm{DP} \rightleftarrows \mathrm{N}_{1} \mathrm{DP}+\mathrm{N}_{2} \mathrm{TP}$}

Les NDP kinases sont peu spécifiques, $\mathrm{N}_{1}$ et $\mathrm{N}_{2}$ peuvent être des dérivés ribo- et désoxyribosylés de bases puriques ou pyrimidiques. La localisation subcellulaire est principalement cytosolique, mais l'enzyme est aussi présente dans les mitochondries et les noyaux, et associée aux membranes plasmiques [7]. Des activités NDP kinase associées à une ADN polymérase [8], à une ribonucléotide réductase [9] et à des protéines liant le GTP (voir plus loin) ont été décrites. Le manque de spécificité et une très forte activité moléculaire qui les rendaient peu susceptibles d'être la cible de régulations fines, expliquent peut-être le relatif désintérêt constaté jusqu'à ces dernières années pour l'étude de ces enzymes.

Des données récentes ont montré que l'enzyme purifiée à partir de globules rouges humains est un hexamère formé de deux types de monomères (A et $\mathrm{B})$ de séquences très homologues (88\% d'identité) [10]. Le mélange des sous-unités purifiées aboutit à l'association des deux types de monomères en proportions variables [10]. Différents types d'hexamères sont ainsi formés (A6, A5B $1, \ldots$ A1B5, B6) qui sont tous actifs et forment une famille d'isoenzymes comparable à celle trouvée dans les extraits cellulaires. L'existence de ces isozymes pourrait refléter des propriétés ou des localisations subcellulaires différentes, associées à des fonctions physiologiques différentes pour l'instant inconnues.

\section{NDP kinase \\ de Dictyostelium discoideum et séquences apparentées}

L'ambibe Diclyostelium discoideum est un organisme modèle pour l'étude du chimiotactisme et de la différenciation cellulaire [11] particulièrement adapté à l'étude des systèmes de transmission des signaux transmembranaires (récepteurs, protéines $G$, etc.) qui $m / s n^{\circ} 5$, vol. 8, mai 92

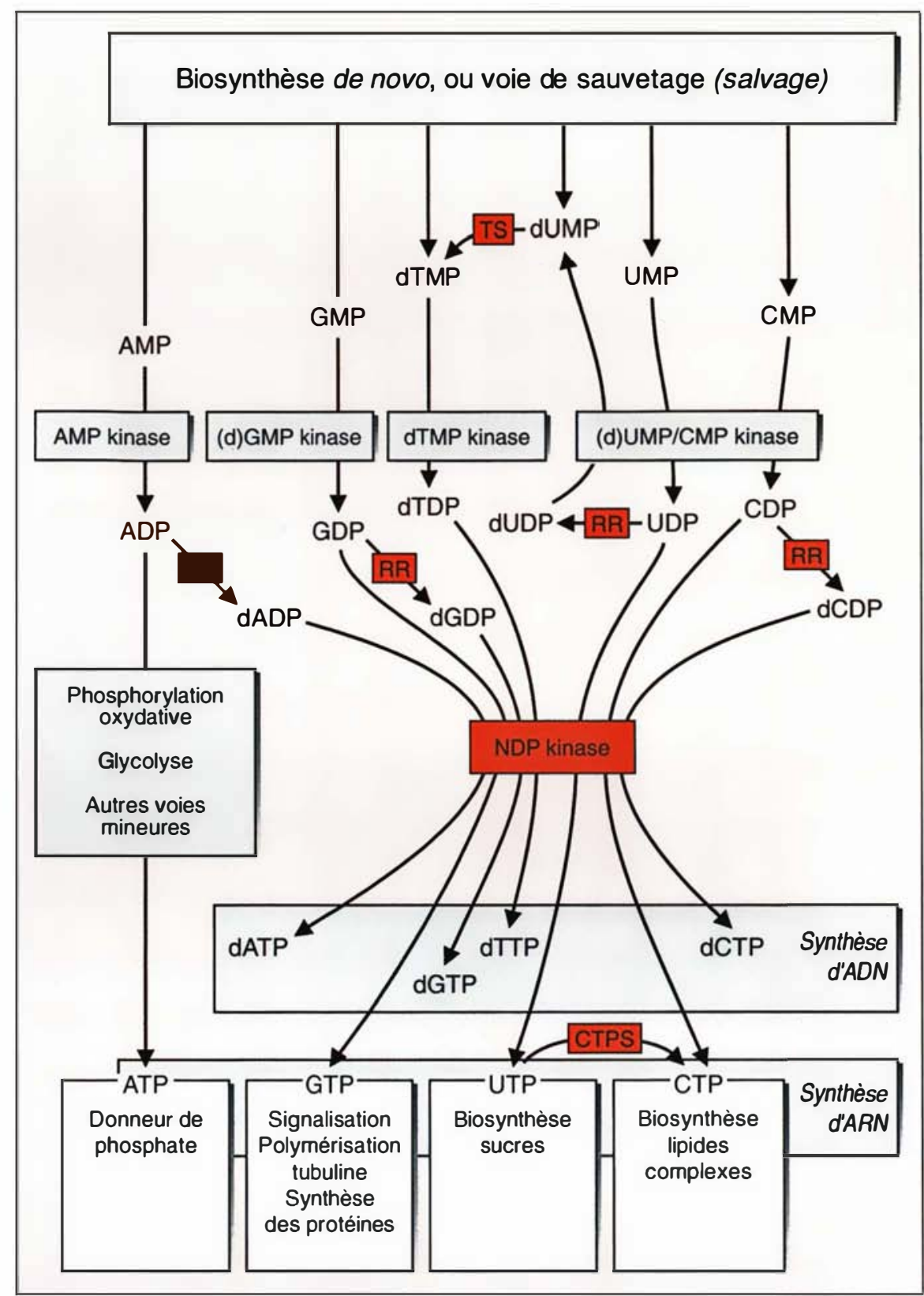

Figure 1. Rôle de la NDP kinase dans la synthèse des nucléotides. La phosphorylation des nucléosides diphosphates est catalysée par une seule enzyme, la NDP kinase, alors que celle des nucléosides monophosphates est due à plusieurs enzymes spécifiques de la base. La réaction catalysée par la NDP kinase est réversible, mais dans le contexte du métabolisme cellulaire, c'est l'ATP qui est le donneur de phosphate préférentiel pour la phosphorylation des nucléosides diphosphates non adényliques. Des voies mineures (comme par exemple la phosphorylation du GDP dans les mitochondries) ne sont pas mentionnées dans ce schéma. RR : ribonucléotide réductase; TS : thymidylate synthase; CTPS; cytidine triphosphate synthase. 


\section{RÉFÉRENCES}

1. Rosengard AM, Krutzsch HC, Shearn A, et al. Reduced Nm23/Awd protein in tumor metastasis and aberrant Drosophila development. Nature 1989 ; 342 : 177-80.

2. Lacombe ML, Wallet V, Troll H, Véron M. Functional cloning of a nucleoside diphosphate kinase from Dictyostelium discoideum. J Biol Chem 1990 ; 265 : 10012-8.

3. Wallet V, Mutzel $\mathrm{R}$, Troll $\mathrm{H}$, et al. Dictyostelium nucleoside diphosphate kinase highly homologous to Nm23 and Awd proteins involved in mammalian tumor metastasis and Drosophila development. J Natl Cancer Inst 1990; 82 : 1199-201.

4. Lacombe ML, Jakobs KH. Nucleoside diphosphate kinase as potential new targets for control of development and cancer. Trends Pharmacol Sci $1992 ; 13$ : 46-8.

5. Munoz-Dorado J, Inouye $\mathrm{M}$, Inouye $\mathrm{S}$. Nucleoside diphosphatase kinase from Myxococcus xanthus. I. Cloning and sequencing of the gene. J Biol Chem 1990 ; 265 : 2702-6.

6. Kimura $\mathbf{N}$, Shimada $\mathrm{N}$, Nomura $\mathrm{K}$, Watanabe $\mathrm{K}$. Isolation and characterization of a cDNA clone encoding rat nucleoside diphosphate kinase. J Biol Chem 1990 ; 265 : 15744-9.

7. Agarwal RP, Robinson B, Parks RE Jr. Nucleoside diphosphate kinase from human erythrocytes. Meth Enzymol 1978 ; 51 : 376-86.

8. Miller LK, Wells RD. Nucleoside diphosphokinase activity associated with DNA polymerases. Proc Natl Acad Sci USA 1971 ; 68 : 2298-302.

9. Von Döbeln U. Nucleoside diphosphate kinase activity associated with ribonucleotide reductase. Biochem Biophys Res Commun $1976 ; 72: 1160-7$

10. Gilles AM, Presecan E, Vonica A, Lascu I. Nucleoside diphosphate kinase from human erythrocytes. Structural characterization of the two polypeptide chains responsible for heterogeneity of the hexameric enzyme. J Biol Chem 1991; 266 : 8784-9.

11. Devreotes P. Dictyostelium discoideum : a model system for cell-cell interaction in development. Science 1989 ; 245 : 1054-8.

12. Dumas $C$, Lebras $G$, Wallet V, Lacombe ML, Véron $M$, Janin J. Crystallization and prelimary X-ray diffraction studes of nucleoside diphosphate kinase from Diclyostelium discoideum. J Mol Biol 1991 ; 217: 239-40.

13. Steeg PS, Bevilacqua G, Kopper L, et al. Evidence for a novel gene associated with low tumor metastasic potential. J Natl Cancer Inst $1988 ; 80: 200-4$

14. Biggs J, Hersperger E, Steeg PS, Liotta LA, Shearn A. A Drosophila gene that is homologous to a mammalian gene associated with tumor metastasis codes for a nucleoside diphosphate kinase. Cell 1990 ; 63 : 993-40.

15. Stahl JA, Leone A, Rosengard AM, Porter L, Richter King C, Steeg PS. Identification of a second human nm23 gene, nm23-H2. Cancer Res 1991; 51 : 445-9.

16. Leone A, McBride WO, Wang MG, et al. Somatic allelic deletion of $\mathrm{nm} 23$ in human cancer. Cancer Res 1991; 51 : 2490-3.

17. Hama $\mathrm{H}$, Almaula $\mathrm{N}$, Lerner $\mathrm{CG}$ Inouye S, Inouye M. Nucleoside diphosphate kinase from Escherichia coli; its overproduction and sequence comparison with eukaryotic enzymes. Gene 1991 ; 105 : 31-6.

18. Leone A, Flatow U, Richter King C et al. Reduced tumor incidence, metastatic potential, and cytokine responsiveness of Nm23 transfected melanoma cells. Cell $1991 ; 65: 25-35$.

19. Lacombe ML, Sastre-Garau X, Lascu I, Wallet V, Thiery JP, Véron M. Overexpression of nucleoside diphosphate kinase (Nm23) in solid tumours. Eur $J$ Cancer $1991 ; 27$ : 1302-7.

20. Sastre-Garau X, Lacombe ML, Jouve M, Véron M, Magdelenat $H$. Nucleoside diphosphate kinase/Nm23 expression in breast cancer: lack of correlation with lymph-node metastasis. Int J Cancer 1992 ; $50: 533-8$

21. Barnes R, Massood S, Barker E, et al. Low $\mathrm{Nm} 23$ protein expression in infiltrating ductal breast carcinomas correlates with reduced patient survival. Am J Path 1991 ; 139 : 245-9.

22. Hennessy C, Henry JA, May FEB Westley BR, Angus B, Lennard TWJ. Expression of the antimetastatic gene $n m 23$ in human breast cancer : an association with good prognosis. J Natl Cancer Inst 1991; 83 281-5.

23. Francis B, Overmeyer J, John W Marshall E, Haley B. Prevalence of nucleoside diphosphate kinase autophosphorylation in human colon carcinoma versus norma colon homogenates. Mol Carcinog 1989 ; 2 : 168-78.

24. Haut M, Steeg PS, Willson JKV, Mar kowitz SD. Induction of $n m 23$ expression in human colonic neoplasms and equal expression in colon of high and low metastatic potential. I Natl Cancer Inst 1991; 83 712-6.

25. Hailat N, Keim DR, Melhem RF, et al. High levels of $\mathrm{p} 19 / \mathrm{nm} 23$ protein in neuro blastoma are associated with advanced stage disease and with N-myc gene amplification $J$ Clin Invest 1991; $88: 341-5$ sont très similaires à ceux décrits chez les mammifères.

L'ADNc codant pour la NDP kinase de Dictyostelium discoideum a été isolé par criblage fonctionnel d'une banque d'expression construite dans le phage $\lambda \mathrm{gt} 11$. L'incubation des filtres répliqués en présence de $\left[{ }^{35} \mathrm{~S}\right] \mathrm{GTP} \gamma \mathrm{S}$ a permis de détecter l'intermédiaire réactionnel phosphorylé marqué par le thiophosphate [2]* . Bien que le niveau de l'ARNm soit modulé au cours du développement, avec un niveau très élevé pendant la phase de prolifération végétative, décroissant rapidement après la mise en carence [3], le niveau de la protéine et son activité restent relativement stables. L'enzyme recombinante de Dictyostelium a été cristallisée [12] et la résolution de la structure tridimentionnelle est en cours dans le laboratoire de J. Janin (Orsay, France). La détermination de la séquence de Dictyostelium, première séquence d'une enzyme de ce type, obtenue chez un eucaryote, a montré qu'elle présentait un haut degré de similitude $(60 \%$ d'identité) avec celles de deux protéines de fonction alors inconnue, Awd et $\mathrm{Nm} 23$ [1]. Le gène $n m 23$ (non metastatic clone 23) a été proposé comme un supresseur du potentiel métastatique de cellules tumorales $[1,13]$ et le gène awd (abnormal wing disc) est nécessaire au développement de la larve de drosophile. En effet, une délétion de ce gène induit des altérations majeures des disques imaginaux des ailes et des yeux/antennes ainsi que de certaines structures du cerveau et des ovaires qui aboutissent à la mort des larves au troisième stade [14]. Le produit d'un deuxième gène d'origine humaine, $n m 23-H 2$ très similaire au gène $n m 23$ initialement décrit et appelé dorénavant $n m 23-H 1$, a également été isolé [15]. Le gène nm23-H1 a été localisé sur le bras long du chromosome $17 \quad$ (17q21; [16]). Les séquences des deux monomètres $A$ et $B$ de la NDP kinase purifiée à partir d'érythrocytes humains [10] sont identiques aux

* Le criblage par expression d'une banque en phage igt 11 consiste a faire synthétiser, par les bactéries de chaque clone, la protéine pour laquelle code l'ADNc inséré sous la forme d'un gène hybride dans le phage $\lambda g t$ 11. Cette protéine est alors transférée sur un filtre et est reconnue soit par son antigénicité, soit, ici, par sa capacité a fixer un ligand radioactif, analogue non hydrolysable du GTP. 


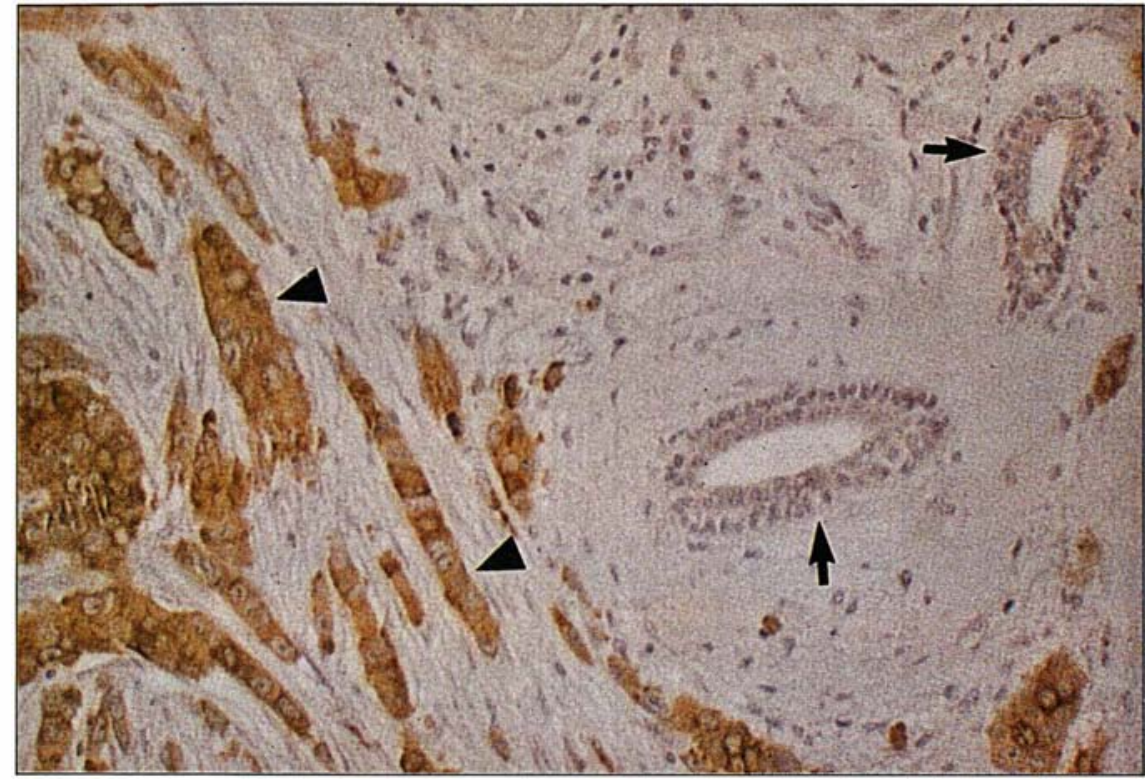

Figure 2. Surexpression de la NDP kinase dans un carcinome intracanalaire infiltrant du sein. $(4)$ : cellules tumorales. $(\rightarrow)$ canaux normaux.

séquences des produits des gènes $n m 23-H 1$ et $n m 23-H 2$, ce qui établit formellement l'identité de $\mathrm{Nm} 23$ comme une NDP kinase. Cette fonction a également été démontrée directement pour la protéine Awd [14]. Des séquences de NDP kinases ont été obtenues chez différents organismes, procaryotes comme Myxococcus xanthus [5] et $E$. coli [17] ou eucaryotes comme le rat [6]. Ces différentes séquences sont très conservées puisqu'il existe $43 \%$ de résidus identiques entre l'enzyme de $E$. coli et l'enzyme humaine.

\section{NDP kinases et prolifération tumorale}

L'ADNc correspondant au gène nm23 a été isolé par Steeg et al. [13] par criblage différentiel de banque d'ADNc avec des sondes ARNm isolées de lignées de potentiel métastatique variable provenant d'un mélanome murin (K1735). L'expression de ce gène est forte dans des lignées peu agressives, alors qu'elle se trouve diminuée dans des lignées à fort potentiel métastatique. Des expériences de transfection de lignées K1735 très agressives avec le gène $n m 23$ murin montrent une diminution de leur potentiel métastatique corrélée à une plus forte expression du produit $\mathrm{m} / \mathrm{s} n^{\circ}$ 5, vol. 8, mai 92 confirmé l'augmentation du niveau d'expression de la NDP kinase plus importante dans les tumeurs malignes que dans les lésions bénignes. Cependant, aucune corrélation n'a été observée entre le taux d'expression de l'enzyme et le risque d'extension métastatique (degré d'envahissement ganglionnaire axillaire) et d'autres marqueurs pronostiques [20]. Nos résultats sont donc en contradiction avec d'autres études sur les tumeurs du sein $[21,22]$ qui montrent une corrélation entre une forte expression de $\mathrm{Nm} 23$ et un bon taux de survie des patients. Cette contradiction est peut-être due à des différences dans l'évaluation de l'expression de la NDP kinase (activité enzymatique, utilisation d'anticorps, ou mesure du taux d'ARN messager) et dans le choix du critère d'évaluation du potentiel métastatique (nombre de ganglions envahis ou taux de survie des patients).

Il apparaît donc que la relation entre un faible taux de NDP kinase et un fort potentiel métastatique n'est observée finalement que dans des séries relativement limitées de tumeurs du sein et seulement dans certains modèles de lignées cellulaires. En revanche, on observe systématiquement dans les tumeurs malignes et certaines tumeurs bénignes, une augmentation du taux de NDP kinase par rapport aux tissus normaux, quoique dans des proportions variables [19, 20, 23, 24]. Ainsi, dans des cancers du côlon, une forte augmentation de la NDP kinase est observée sans corrélation avec le potentiel métastatique [23, 24]. Dans des cas de neuroblastomes, une hyperexpression de la NDP kinase A est observée, d'autant plus forte que les stades de la tumeur sont plus agressifs [25]. L'augmentation du taux de NDP kinase pourrait refléter l'état de prolifération cellulaire puisqu'une étude sur des lymphocytes montre que la NDP kinase augmente après stimulation des cellules [26]. Un travail déjà ancien sur la régénération hépatique montre que celle-ci s'accompagne aussi d'une augmentation de l'activité NDP kinase [27]. Quel peut être le rôle des NDP kinases dans le contrôle de la prolifération tumorale ou le développement? On peut s'attendre à une augmenta- 


\section{RÉFÉRENCES}

26. Keim D, Hailat N, Melhem R, et al. Proliferation-related expression of p19/Nm23 nucleoside diphosphate kinase. J Clin Invest 1992 (sous presse).

27. Chiga M, Oda A, Holtzer RL. The activities of certain nucleoside diphosphokinases of normal and regenerating rat liver. Arch Biochem Biophys 1963 ; 103 : 366-70.

28. Jackson RC, Lui MS, Boritzki TJ, Morris HP, Weber G. Purine and pyrimidine nucleotide patterns of normal, differentiating, and regenerating liver and hepatomas in rats. Cancer Res $1980 ; 40$ : 1286-91.

29. Tompson FM, Atkinson DE. Response of nucleoside diphosphate kinase to the adenylate energy charge. Biochem Biophys Res Commun 1971; 45 : 1581-5.

30. Bourne HR, Sanders DA, McCormick F. The GTPase superfamily: conserved structure and molecular mechanism. Nature $1991 ; 349$ : 117-27.

31. Penningroth SM, Kirschner MW Nucleotide binding and phosphorylation in microtubule assembly in vitro. I Mol Bio $1977 ; 115: 643-73$.

32. Kimura N, Nagata N. Mechanism of glucagon stimulation of adenylate cyclase in the presence of GDP in rat liver plasma membranes. I Biol Chem 1979 ; 254 3451-7.

33. Randazzo PA, Northup JK, Kahn RA Activation of a small GTP-binding protein by nucleoside diphosphate kinase. Science $1991 ; 254: 850-3$.

34. Teng DH, Engele CM, Venkatesh TR. A product of the prune locus of Drosophila is similar to mammalian GTPases-activating protein. Nature 1991 ; 353 : 437-40.

35. Ohtsuki K, Yokoyama M. Direct activation of guanine nucleotide binding proteins through a high-energy phosphate transfer by nucleoside-diphosphate kinase. Biochem Biophys Res Commun 1987 ; 148 : 300-7.

36. Otero AS. Transphosphorylation and G protein activation. Biochem Pharmacol 1990 ; 39 : 1399-404

37. Kikkawa S, Takahashi K, Takahashi $\mathrm{K}$, et al. Conversion of GDP into GTP by nucleoside diphosphate kinase on the GTPbinding proteins. J Biol Chem $1990 ; 265$ : 21536-40.

38. Kikkawa S, Takahashi $\mathrm{K}$, Takahashi $\mathrm{K}$, et al. Conversion of GDP into GTP by nucleoside diphosphate kinase on the GTPbinding proteins. Correction. $J$ Biol Chem
39. Wieland T, Bremerich J, Gierschik P Jakobs KH. Contribution of nucleoside diphosphokinase to guanine nucleotide regulation of agonist binding to formyl peptide receptors. Eur J Pharmacol 1991; 208 : 17-23

40. Kimura N, Shimada N. Differential susceptibility to GTP from added GDP via membrane-associated nucleoside diphosphate kinase of GTP-sensitive adenylate cyclase achieved by hormone and cholera toxin. Biochem Biophys Res Commun 1985; 131: 199-206.

41. Walton GM, Gill GN. Nucleotide regulation of a eukaryotic protein synthesis ini tiation complex. Biochim Biophys Acta 1975 ; 390 : 231.45

42. Islam K, Burns RG. Microtubules and nucleoside diphosphate kinase. Nucleoside diphosphate kinase binds to co-purifying contaminants rather than to microtubule proteins. Biochem J 1985 ; 232 : 651-6.

43. Ohtsuki $\mathrm{K}$, Ikeuchi $\mathrm{T}$, Yokoyama $\mathrm{M}$ Characterization of nucleoside-diphosphate kinase-associated guanine nucleotide-binding proteins from HeLa S3 cells. Biochim Biophys Acta 1986 ; 882 : 322-30.

44. Kimura N, Shimada N. Evidence for complex formation between GTP binding protein (Gs) and membrane-associated nucleoside diphosphate kinase. Biochem Biophys Res Commun 1990 ; 168 : 99-106.

45. Huitorel $P$, Simon C, Pantaloni D. Nucleoside diphosphate kinase from brain. Purification and effect on microtubule assembly in vitro. Eur J Biochem $1984 ; 144$ 233-41

46. Bischoff FR, Ponstingl H. Catalysis of guanine nucleotide exchange on $\mathrm{R}$ an by the mitotic regulator RCC1. Nature $1991 ; 354$ 80-2.

47. Biggs J, Tripoulas $\mathrm{N}$, Hersperger $\mathrm{E}$, Dearolf CR, Shearn A. Analysis of the lethal interaction between the prune and Killer of prune mutations of Drosophila. Genes Dev $1988 ; 2$ : 1333-43.

48. Lascu I, Chaffotte A, Limbourg Bouchon B, Véron M. A Pro/Ser substitution in nucleoside diphosphate kinase of Drosophila melanogaster (mutation Killer of prune) affects stability but not catalytic efficiency of the enzyme. J Biol Chem 1992 (sous presse).

49. Yatani A, Quilliam LA, Brown AM Bokoch GM. Rap1A antagonizes the ability of Ras and Ras-Gap to inhibit muscarinic K + Channels. J Biol Chem 1990 ; 266 22222-6.

50. Woods DF, Bryant PJ. The discs-large tumor suppressor gene of drosophila encodes a guanylate kinase homolog localized a septate junctions. Cell 1991; 66 : 451-64. 51. Okabe-Kado J, Kasukabe T, Honma Y, Hayashi M, Hentzel WJ, Hozumi M. Identity of a differentiation inhibiting factor for mouse myeloid leukemia cells with Nm23/nucleoside diphosphate kinase. Biochem Biophys Res Commun $1992 ; 182$ : 987-94. tion de l'activité de l'enzyme lors d'une synthèse accrue des acides nucléiques, $\mathrm{ADN}$ et $\mathrm{ARN}$; d'ailleurs, plusicurs enzymes intervenant dans les voies de biosynthèse des nucléotides sont plus actives dans les tumeurs [28]. Il a également été suggéré que les NDP kinases, en tant qu'enzymes limitantes dans la synthèse des nucléosides triphosphates, puissent agir sur la croissance de la cellule par le contrôle de son équilibre énergétique et de son stock de nucléosides triphosphates, une dérégulation favorisant une prolifération anarchique des cellules [29]. Enfin, ces enzymes pourraient ćgalement intervenir plus spécifiquement en tant que "GTP synthase " dans l'activation des protéines liant les nucléotides guanyliques.

\section{NDP kinases et protéines liant le GTP}

La possibilité d'un rôle des NDP kinases dans l'activation des protéines liant les nucléotides guanyliques, aussi appelées GTPascs [30], a été formuléc depuis plus de dix ans pour la polymérisation des microtubules [31] et pour l'activation $\mathrm{du}$ système adénylate cyclase [32]. Longtemps controverséc et considéréc comme hérétique par la communauté scientifique, elle a recu très récemment des supports expérimentaux supplémentaires [33, 34]. Les GTPases jouent un rôle crucial dans toute une série de processus comme la synthèse protéique, la transmission des signaux transmembranaires, la prolifération, la différenciation cellulairc ou la transformation maligne [30]. Malgré leur très grande diversité, l'activation des protéines liant les nucléotides guanyliques est contrôléc par un mécanisme commun : lc passage de la forme inactivée liée au GDP à la forme activée liée au GTP fait intervenir un facteur d'échange. Le retour à la forme inactive s'cffectue par hydrolyse du GTP lié, duc à l'activité GTPase intrinsèque de la protéine, favorisée ou non par une GTPase activating protein, GAP [30]. Il a été proposé que les NDP kinases puissent intervenir dans ce processus (figure 3) en synthétisant lc GTP à proximité des GTPases et en favorisant le transfert du nucléotide par un 


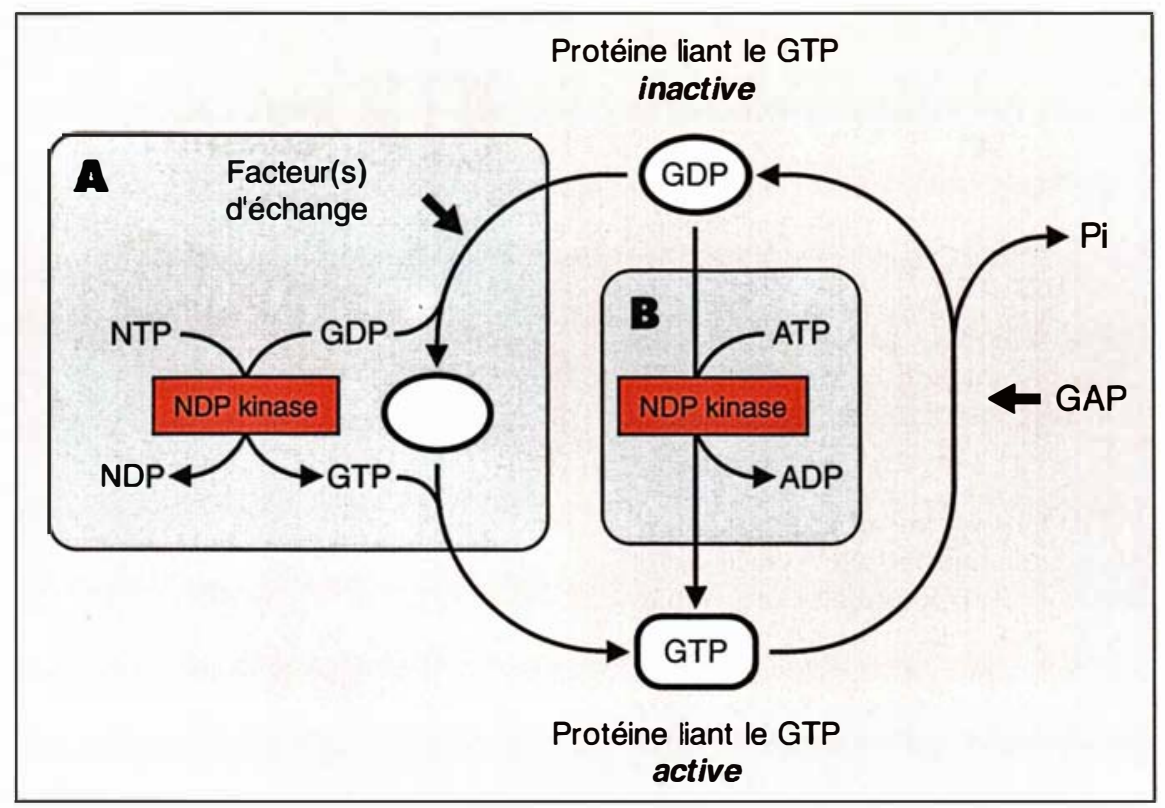

Figure 3. Schéma impliquant les NDP kinases dans l'activation des protéines liant les nucléotides guanyliques. (A). Échange du nucléotide lié et synthèse du GTP à proximité. (B) Phosphorylation in situ du GDP lié.

processus de tunnélisation (channeling) ou en phosphorylant in situ le GDP lié sans dissociation du nucléotide et donc, dans cette dernière hypothèse, sans nécessité d'un facteur d'échange [35-39]. Compte tenu de la forte concentration intracellulaire de GTP (de 0,1 à $1 \mathrm{mM}$ ), les modèles classiques d'activation des protéines liant le GTP considèrent que la disponibilité du nucléotide n'est pas un facteur limitant. Néanmoins, in vivo, les cycles d'activation des GTPases et d'hydrolyse du GTP peuvent être extrêmement rapides [36], ce qui est susceptible d'augmenter considérablement la concentration locale de GDP. Dans le cas d'une compartimentalisation des pools de nucléotides, une synthèse très efficace de GTP paraît être nécessaire au maintien de la stimulation. Dans ce sens, des données cinétiques sur l'activation des systèmes adénylate cyclases suggèrent que le GTP synthétisé localement pourrait être plus efficace pour l'activation que le GTP exogène [39, 40].

Une association physique entre NDP kinases et protéines liant le GTP a été rapportée dans un certain nombre de cas, comme celui du facteur d'initiation eIF2 [41], de petites protéines liant le GTP [43], de la sous- unité $\alpha$ de la protéine Gs de foie de rat [44] et des microtubules [14, 31, 42]. Le réseau microtubulaire de cellules de drosophile peut être décoré par des anticorps anti-NDP kinases et les chromosomes de larves homozygotes pour la mutation nulle du gène awd ressemblent à des chromosomes de cellules traitées à la colchicine [14], suggérant une désorganisation du système microtubulaire.

Cependant, l'interaction microtubules-NDP kinase ne semble pas directe $[42,45]$, et aucune indication d'un effet de la NDP kinase sur la polymérisation de la tubuline in vitro n'a pu être trouvée (R. Melki, M.F. Carlier, communication personnelle). Il est aussi possible que l'aspect des chromosomes chez les mutants soit dû à un défaut d'activation d'une petite protéine $G$, Ran, indispensable à la condensation des chromosomes [46].

Un certain nombre de données cinétiques d'activation et d'inhibition de systèmes de transduction de signaux transmembranaires suggèrent l'intervention de NDP kinases dans des processus réglés par des grandes protéines $\mathrm{G}$ hétérotrimériques. C'est le cas de l'activation muscarinique des canaux potassiques de cellules cardia- ques de grenouille [36], de la stimulation de la NADPH oxydase, de la régulation de l'affinité des récepteurs pour le peptide FMLP* dans des cellules HL60 et de l'inhibition ou l'activation de systèmes adénylate cyclases (cités dans [39]).

La démonstration d'un rôle des NDP kinases dans l'activation des GTP ases se heurte à des difficultés expérimentales. Ainsi, pour tester l'hypothèse d'une phosphorylation in situ du GDP lié à la protéine, il est essentiel de s'assurer qu'il n'y a pas de dissociation du nucléoside diphosphate, substrat potentiel de la NDP kinase. A cet égard, la démonstration d'une phosphorylation in situ du GDP lié aux sous-unités $\alpha$ des protéines Gs et Go hétérotrimériques [37] est apparue critiquable [38]. Cependant, il a été montré très récemment par des expériences utilisant des protéines purifiées recombinantes que le GDP lié à ARF ( $A D P$ ribosylating factor) pouvait être phosphorylé in situ par l'addition de NDP kinase sans dissociation du nucléotide [33]. Il n'est pas connu à ce jour si d'autres complexes GDPGTPases peuvent être " substrats " de NDP kinases.

Un autre argument en faveur d'une interaction physiologique entre NDP kinase et systèmes des protéines liant le GTP vient de l'analyse de mutants de drosophile. En effet, une mutation ponctuelle du gène awd appelée $k p n$ (killer of prune) est une mutation létale conditionnelle dominante qui, seule, ne présente pas de phénotype particulier, mais est létale en association avec la mutation prune, ce qui suggère une interaction fonctionnelle entre les produits de ces deux gènes [47, 48]: La séquence de l'ADNc correspondant au locus prune vient d'être déterminée [34] et correspond à une protéine qui présente des similarités avec le domaine catalytique d'une GAP. Ainsi une NDP kinase pourrait interagir avec une protéine GAP pour le contrôle de l'activité d'une petite protéine $G$ de type Ras. Il est possible que les produits de ces deux gènes interagissent au sein d'un réseau complexe comme les systèmes GAP/Ras/Rap qui ont été décrits pour la régulation des canaux potas-

- FMLP : Formyl-Met-Leu-Phe, peptide chimiotactique attirant les neutrophiles. 
siques [49]. Dans cette perspectivc, la possibilité d'unc intcraction NDP kinasc/GAP dans les signalosomes, complexes multiprotéiques impliqués dans la transmission des signaux mitotiques, devrait ĉtre testéc.

En conclusion, il apparaît que les NIPP kinases pourraicnt joucr un rôle majcur dans le contrôle de l'activité des protéines liant le GTP. Compte tenu de la multiplicité des cibles potenticlles des NDP kinases, des altérations dans l'expression de ces enzymes devraient avoir des effets pléiotropes sur le fonctionnement de la cellulc. L'activation pourrait être directe et, dans certains cas, courtcircuiter les protéines d'échange, comme le montrent les résultats relatifs à la protéinc $\mathrm{ARF}$, ou indirectc par modification du rapport des concentrations GTP/GDP. Des résultats très récents indiquent que des modifications locales de ce rapport peuvent avoir unc incidence sur la prolifération cellulaire. Ainsi, unc mutation dans le gène $d l g$ codant pour unc protéine homologue à unc guanylate kinasc (enzyme catalysant la synthèse de GDP) est responsable de la formation de tumeurs chez la drosophile. Cettc mutation induirait un défaut dans la synthèse localc de cc nucléotide, d'où unc altération du rapport GTP/GDP et unc activation des GTPases impliquécs dans la prolifération des cellules épithéliales de la mouche [50].

La relation entre unc faible expression de NDP kinase et le potenticl métastatique des cellules tumorales, qui a récemment focalisé l'attention șur cette enzyme, nc présente pas le caractèrc général qu'on aurait voulu lui attribucr. Les données montrent que les cellules tumorales présentent un fort taux de NDP kinases qui pourrait scrvir de marqueur, au moins dans certains types de cancers, cc qui mériterait certainement des études cliniques approfondics. La très récentc identification, comme unc forme monomérique ou dimérique de NIP kinasc, d'un facteur cxcrété, inhibitcur de la différenciation ouvre des perspectives absolument nouvelles [51]. Lc développement d'outils comme les anticorps ou les sondes spécifiques vont permettre, dans un avenir proche, de préciser le rôle physiologique de cettc classe d'enzy- mes dans le développement, la prolifération et éventuellement la différenciation de cellules normales ou tumorales

\section{Summary}

NDP kinases, development and cancer : an action through GTP binding proteins?

NDP kinases are ubiquitous enzymes essential for the synthesis of nucleoside triphosphates other than ATP. The recent identification as NDP kinases, of the Awd protein crucial for drosophila development and of the $\mathrm{Nm} 23$ protein involved in mammalian tumor malignancy indicates that these enzymes could be involved in the control of development and cancer. The observation of an inverse corrclation between the expression of $\mathrm{Nm} 23$ and the metastatic potential appears to be restricted to some studies on breast tumors and to some cell lines. Recent data point to an over-expression of NDP kinases in solid tumors as compared to corresponding normal tissues, probably related to the proliferative state of the tumoral cells. The mechanism of this overexpression is unknown but could be related to the proposed role of NDP kinases in GTPases activation which could act either by direct phosphorylation of the bound GDP, or by synthetizing GTP within complexes involved in GTP channeling.

\section{TIRÉS A PART}

M.-I.. I.acombe. 